\title{
Evaluación del rendimiento de redes ópticas para aplicaciones de telemedicina en ambientes simulados
}

\section{Performance evaluation of optical networks for telemedicine applications in simulated enviroments}

\section{Camilo Andrés Lemus Bernal}

Ingeniero en Telecomunicaciones. Investigador de la Universidad Militar Nueva Granada. Bogotá, Colombia. Contacto: securityresearch@unimilitar.edu.co

\section{Edith Paola Estupiñán Cuesta}

Ingeniera en Telecomunicaciones. Asistente de investigación de la Universidad Militar Nueva Granada. Bogotá, Colombia.

Contacto: securityinvgroup@unimilitar.edu.co

\section{Edward Paul Guillén Pinto}

Ingeniero Electrónico, magister en Teleinformática. Docente e Investigador de la Universidad Militar Nueva Granada. Bogotá, Colombia.

Contacto:gissic@unimilitar.edu.co

Palabras clave: calidad de servicio, redes ópticas, simulación, sistemas de información médica, telemedicina.

Key words: quality of service, optical networks, simulation, medical information systems, telemedicine.

\section{RESUMEN}

Las redes de telemedicina permiten la prestación de ciertos servicios médicos de manera remota. Cada servicio demanda ciertos requerimientos de red para su correcto funcionamiento. En la actualidad, la Universidad Militar Nueva Granada no cuenta con una red experimental de telemedicina donde puedan realizarse algunos procedimientos médicos con fines académicos, por esta razón, se propone el diseño de una red óptica para servicios de telemedicina entre la sede central de la Universidad Militar Nueva Granada y la sede de Medicina, ubicada en el Hospital Militar Central. Dentro de la propuesta de red de telemedicina se evalúa el rendimiento de acuerdo a los requerimientos de las aplicaciones de la telecardiología y el registro clínico electrónico; finalmente, se 
compara el rendimiento de la red óptica, con los valores establecidos por las normas y estándares internacionales.

\section{ABSTRACT}

Telemedicine networks allow remote delivery of medical services. Each service uses some applications, such as videoconference or audio-conference in order to conduct medical procedures. These services imply certain minimum requirements to

\section{INTRODUCCIÓN}

La telemedicina permite realizar algunos procedimientos médicos de manera remota, como exámenes de diagnóstico y supervisión de tratamientos, utilizando recursos teleinformáticos [1]. La American Telemedicine Association define la telemedicina como "la prestación de cualquier servicio de salud o la transmisión de información médica empleando sistemas y tecnologías de comunicación". Con la práctica de la telemedicina es posible obtener beneficios en el campo de la salud, como la posibilidad de brindar asistencia médica en zonas de difícil acceso prestar nuevos servicios clínicos y mejorar la calidad de los servicios existentes en centros de salud donde no se cuenta con la presencia de médicos especialistas [2].

Las aplicaciones necesarias para prestar los servicios de telemedicina, como la teleconferencia y la videoconferencia, entre otras, demandan ciertos requerimientos mínimos de las redes, como la capacidad de canal requerida o los parámetros de calidad de servicio, para garantizar la disponibilidad de los servicios y el correcto funcionamiento de las aplicaciones para los usuarios finales. ensure appropriate performance. At present, Universidad Militar Nueva Granada has no such telemedicine network for academic purposes, where students (medicine students and researchers) can deploy some e-health services. In this paper, we propose an optical network for telemedicine services intended to connect two of the headquarters of Universidad Militar Nueva Granada. Then we evaluate the performance of the network according to application requirements. Finally, we compare the performance of the optical network and the values stated by international standards.

$*$

Las redes actuales de telemedicina permiten ofrecer distintos servicios sobre las aplicaciones nombradas anteriormente, sin embrago, debido al tipo de información que se utiliza en medicina, que es de tipo confidencial y de carácter prioritario en las redes de información, es necesario establecer canales de información que permitan brindar la seguridad, confiabilidad y disponibilidad necesarias para los servicios médicos.

En este artículo, se presenta la evaluación del rendimiento de una red óptica, establecida para servicios de telemedicina entre dos sedes de la Universidad Militar Nueva Granada. En la primera parte, se expone el concepto de una red óptica y su funcionamiento, y se da una breve explicación de la clasificación de los servicios de telemedicina. En la segunda parte se hace un resumen de las variables y parámetros de Calidad de Servicio (QoS) que serán evaluados. En la tercera, se muestran y se explican los escenarios a evaluar. En la cuarta sección se muestran los resultados de desempeño y calidad de servicio obtenidos en las simulaciones. Y, finalmente, se exponen las conclusiones conseguidas con base en los resultados obtenidos.

La telemedicina se ha convertido en una herramienta que facilita el acceso a servicios médicos 


\section{investigación}

a las personas que se encuentran en lugares de difícil acceso y que no cuentan con los medios necesarios para ser atendidos en los centros de salud. La creciente demanda de servicios médicos, en zonas apartadas, genera la necesidad de evaluar el rendimiento de varios tipos de redes diferentes a las usadas tradicionalmente para establecer distintas alternativas para la transmisión de datos médicos. Una de estas son las redes ópticas, las cuales pueden ofrecer beneficios importantes para el transporte de datos, en comparación con las redes Ethernet tradicionales.

\section{METODOLOGÍA}

La investigación se clasifica, según su finalidad, en una investigación aplicada y la metodología definida se fundamenta en características del método científico y del tipo de investigación. A continuación se detalla brevemente cada etapa $\mathrm{y}$, en la figura 1 , se muestra gráficamente las etapas de la metodología.

Etapa I. Revisión documental: en esta etapa inicial y de tipo teórico, se realizó una revisión documental con el fin de analizar las características de las tecnologías para el transporte y conmutación óptica, así mismo, los avances presentados en este tema.

Etapa II. Análisis de redes ópticas: en esta etapa se analizaron las redes ópticas para aplicaciones en telemedicina, este proceso comprendió el análisis de características de redes ópticas e investigación sobre aplicaciones medicas en redes ópticas.

Etapa III. Estudio de servicios de telemedicina y sus requerimientos: se realizó un estudio sobre los servicios convencionales prestados en una red de telemedicina para, posteriormente, seleccionar dos de ellos. Una vez seleccionados se definieron las aplicaciones multimedia de cada servicio y sus parámetros para una eventual simulación.

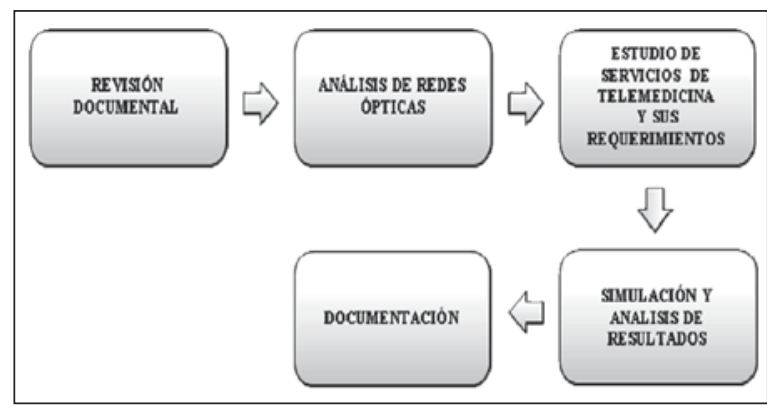

Figura 1. Metodología

Fuente: elaboración propia.

Etapa IV. Simulación y análisis de resultados: se integraron todos los parámetros definidos para soportar los servicios de telemedicina seleccionados sobre la red en un ambiente simulado. Y una vez simulada la red, se evaluó el rendimiento de la misma.

Etapa V: Documentación: esta etapa final se recopilo ordenada y lógicamente los resultados de la investigación.

\section{ANTECEDENTES}

Algunas herramientas de simulación, como NS2 y OPNET, han sido empleadas para el modelamiento de redes de telemedicina, con el fin de evaluar el rendimiento de determinadas aplicaciones en el uso de nuevos modelos y arquitecturas en redes. El desarrollo de ciertos trabajos se ha basado en la optimización de redes existentes mediante la aplicación de diseños de arquitecturas alternas a las usadas actualmente, como las arquitecturas por capas [3], [4], mientras que otros se han basado en la integración de redes existentes de telemedicina con nuevas tecnologías, para garantizar y mejorar la calidad de los servicios de telemedicina [5], [6]. En ambos tipos de trabajos se han empleado herramientas de simulación de redes para evaluar el desempeño de los nuevos modelos de telemedicina. Los resultados han permitido conocer qué tipo de tecnologías se adaptan mejor 
a los servicios de telemedicina y permiten ofrecer parámetros de calidad de servicio adecuados para asegurar la prestación de los servicios sin sacrificar la salud de los pacientes.

\section{REFERENTES TEÓRICOS}

En esta sección se presentan algunos conceptos teóricos que serán utilizados como base para el desarrollo del artículo.

\subsection{Redes ópticas}

Las tecnologías de transmisión en redes ópticas han evolucionado en los últimos años debido al creciente desarrollo de servicios que demandan altas velocidades y calidad en la transmisión de datos, con anchos de banda apropiados. Actualmente, las redes ópticas pueden ofrecer capacidades óptimas para la transmisión de datos multimedia, usando técnicas de transporte y multiplexación.

El funcionamiento de las redes ópticas se encuentra dividido jerárquicamente en un modelo que se

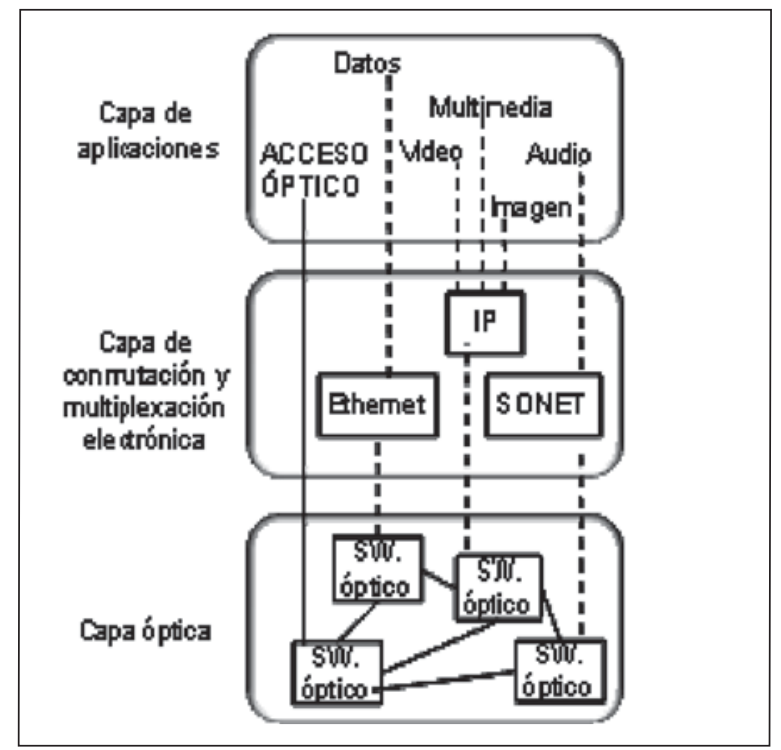

Figura 2. Modelo de arquitectura óptica por capas Fuente: tomada de [7]. compone de tres capas, como se muestra en la figura 2. La capa de aplicación incluye todos los tipos de servicios (voz, video y datos). La capa intermedia comprende la multiplexación, el transporte y la conmutación, con tecnologías electrónicas, en esta capa se pueden encontrar dispositivos como: routers IP, Switches Ethernet, Switches ATM y Switches SONET/SDH. Finalmente, se encuentra la capa óptica, donde se toman las señales obtenidas de la capa intermedia, o capa electrónica, y se modulan luego en portadoras ópticas correspondientes a distintas longitudes de onda. La capa óptica está basada en la tecnología WDM y utiliza switches configurables capaces de conmutar longitudes de onda de forma dinámica [7].

\subsubsection{Elementos de una red óptica}

En las redes ópticas existe una amplia variedad de dispositivos que permiten la transmisión y el transporte de datos. Los dispositivos más importantes son: las fuentes generadoras [8], los amplificadores ópticos [9], los acopladores, los filtros ópticos [10], los multiplexores y demultiplexores [11], los multiplexores de extracción e inserción óptica (OADM), conmutadores [12], multiplexores por división de tiempo ópticos (OTDM) [13], entre otros.

\subsubsection{Tecnologías ópticas de transporte}

SDH es un conjunto jerárquico de estructuras de transporte digital, que está estandarizado para transportar cargas útiles adecuadamente adaptadas sobre redes de fibra óptica. SDH maneja un módulo de transmisión síncrona (STM), definido como la estructura de información utilizada para soportar las conexiones en esta arquitectura, conformada por una parte de carga útil de información y otra llamada tara de sección ( $\mathrm{SOH})$, estos dos campos de información de la STM forman una trama de datos de 2430 octetos que se repiten cada $125 \mathrm{~ms}$ [14], [15]. 


\section{investigación}

SONET al igual que SDH se define como una arquitectura de transporte óptico que, mediante anillos de fibra óptica, permite que datos e información de control sean intercambiados de manera electrónica por los nodos de dicho anillo. Dentro de sus particularidades, SONET está regulada por ANSI y está diseñada para ser usada en países como Estados Unidos, Canadá y Taiwán. Es posible considerar a SONET como un subconjunto de SDH, debido a que SONET habla de su modulo básico de transmisión como un STS-3c o Señal Síncrona de Transporte Nivel 3 Concatenada, coincidiendo su funcionalidad, tamaño y velocidad binaria con los de un modulo básico de transmisión en SDH, STM-1 [16]. La trama básica STS-1 de SONET consiste en 90 columnas y 9 filas de bytes, lo que hacen un total de 810 bytes. Con una duración de $125 \mathrm{~ms}$, lo que permite determinar la capacidad básica de SONET como 51840 bps.

El Modo de Transferencia Asíncrona (ATM) es una tecnología de transporte que proporciona flexibilidad en la transmisión de voz, video y datos. ATM tiene la característica de disponer de una técnica propia para la conmutación de los datos, en la que utiliza unidades de datos de tamaño fijo denominadas celdas [17], [18]. Al contar con un patrón para el tamaño de las unidades de datos, se aumenta la eficiencia en los conmutadores de manera significativa. Además, ATM proporciona un uso eficiente del ancho de banda, ya que brinda la posibilidad de usar tanto una tasa de transmisión constante (por ejemplo para la transmisión de voz), como una tasa de transmisión variable (para la transmisión de datos), utilizando de una forma eficiente el ancho de banda de la red [19] - [22].

\subsection{Servicios de telemedicina}

Generalmente, la telemedicina puede ser vista como un nivel de sofisticación mayor sobre el uso de las tecnologías de comunicación e infor- mación, específicamente en el ámbito de la salud [23]. La telemedicina presta servicios de salud a distancia, mediante la utilización de tecnologías de información y comunicación, las cuales permiten intercambiar datos con el fin de examinar, diagnosticar y tratar un paciente, facilitando el acceso de la población, independientemente del área geográfica donde habiten. Comúnmente, una red de telemedicina cuenta con enlaces de comunicación de baja y alta velocidad. Los enlaces de baja velocidad se usan para la conexión entre los médicos y pacientes con las entidades de salud, y los enlaces de alta velocidad establecen la conexión entre las entidades de salud [24].

Una red de telemedicina debe contar con personal médico capacitado en el manejo de equipos y ejecución de actividades, como el registro de signos vitales, toma de fotografías, videoconferencia y elaboración de registros clínicos electrónicos. Los datos generados por estas actividades deben ser almacenados en una base de datos, donde el

Tabla 1. Servicios y especialidades en Telemedicina

\begin{tabular}{|c|c|}
\hline \multicolumn{2}{|c|}{ Servicios de Telemedicina } \\
\hline Servicios & Especialidades \\
\hline Teleconsulta & Registro Clínico Electrónico (EHR) \\
\hline \multirow{4}{*}{ Tele-Diagnóstico } & Tele-endoscopia \\
\hline & Tele-dermatología \\
\hline & Tele-oftalmología \\
\hline & Tele-otorrinolaringología \\
\hline Tele-Cuidado & Tele-atención \\
\hline \multicolumn{2}{|l|}{ Tele-Educación } \\
\hline \multirow{3}{*}{ Tel-terapia } & Tele-psiquiatría \\
\hline & Tele-fisioterapia \\
\hline & Tele-prescripción \\
\hline \multicolumn{2}{|l|}{ Tele-farmacia } \\
\hline \multirow{3}{*}{ Telemetría } & Tele-radiología \\
\hline & Tele-patología \\
\hline & Telecardiología \\
\hline $\begin{array}{l}\text { Tele-Administra- } \\
\text { ción en Salud }\end{array}$ & \\
\hline
\end{tabular}

Fuente: tomada de [26]. 
especialista tenga acceso a la información del paciente, realizar el análisis de este y finalmente proporcionar su diagnóstico con el fin de que la persona encargada realice el procedimiento adecuado y se tomen las medidas pertinentes con el paciente [25].

Los servicios de telemedicina pueden ser clasificados según el tipo de aplicación y especialidades derivadas de los servicios. En la tabla 1 se observa una descripción de los servicios de telemedicina con sus especialidades de acuerdo a la Organización Panamericana de la Salud OPS/OMS [26].

\section{SERVICIOS A IMPLEMENTAR}

Para efectos de la evaluación del rendimiento de una red óptica para servicios de telemedicina, se han seleccionado los servicios de telemetría y teleconsulta, con sus especialidades telecardiología y registro clínico electrónico. Debido a que se trata de una red experimental, la elección de los servicios se realizó teniendo en cuenta el tipo de información que utilizan, estas dos especialidades se relacionan con aplicaciones de audio/videoconferencia, transferencia y almacenamiento de archivos en bases de datos que permiten una evaluación mayor del rendimiento, ya que involucra varias aplicaciones.

\subsection{Registro clínico electrónico}

Llamado, por su sigla en inglés, Electronic Health Register (EHR). Los registros clínicos electrónicos son definidos como bancos de información médica de pacientes asociados a una entidad de salud particular, que pueden ser almacenados y procesados por computadores. Estos registros incluyen componentes básicos de cada paciente, como: información personal, datos demográficos, registro de progresos médicos, alergias, información acerca de las visitas medicas, medicamentos, signos vitales, antecedentes médicos, historia fa-
Tabla 2. Proceso paso a paso para un EHR

\begin{tabular}{|c|l|}
\hline \multicolumn{2}{|l|}{$\begin{array}{l}\text { Procesos básicos para generar un registro clínico } \\
\text { electrónico }\end{array}$} \\
\hline 1. & Ingreso paciente a la entidad de salud \\
\hline 2. & Identificación del paciente \\
\hline 3. & $\begin{array}{l}\text { Registro de nuevo paciente (diligenciamiento Datos } \\
\text { personales) si es el caso }\end{array}$ \\
\hline 4. & Actualización de datos de paciente (si es necesario) \\
\hline 5. & Registro de incidentes médicos del paciente \\
\hline 6. & $\begin{array}{l}\text { Almacenamiento de registro clínico en la base de } \\
\text { datos }\end{array}$ \\
\hline
\end{tabular}

Fuente: tomada de [27].

miliar, hospitalizaciones, vacunas, datos de laboratorio e informes de radiología [27].

Para una entidad de salud particular, los registros clínicos electrónicos requieren establecer un formato estándar para el almacenamiento de los datos del paciente. Este formato se debe ajustar a los lineamientos particulares de los estándares regulatorios de la salud, como HIPPA y CALDICOTT. Un EHR debe tener la posibilidad de ser modificado, actualizado y almacenado en una base de datos. El equipo primordial para llevar a cabo esta especialidad médica es un computador que permite el almacenamiento y procesamiento de los registros clínicos. La tabla 2 muestra los procedimientos básicos para llevar a cabo un EHR.

\subsection{Telecardiología}

Este servicio se define como la aplicación de la telemedicina a la prevención, diagnóstico y tratamiento de las enfermedades cardiovasculares. Puede realizarse en tiempo real o en diferido, con médicos especialistas cardiólogos para evitar traslados y resolver urgencias [28].

El objetivo del servicio de telecardiología permite la captura de imágenes ECG, para, posteriormente, ser transmitidas y almacenadas en un 


\section{investigación}

punto central. Este tipo de servicios requieren de un servidor de datos que, por medio de un software especializado que permita el envío de información y de almacenamiento a través de la red de comunicaciones hacia estaciones remotas, realice el manejo de las imágenes recibidas.

Los equipos primordiales necesarios para realizar el servicio de telecardiología son: un servidor de datos para el almacenamiento de imágenes, electrocardiograma con salida digital, software especializado, placas metálicas y estetoscopio Digital. En la tabla 3 se muestran los procesos básicos para practicar un servicio de telecardiología.

Tabla 3. Procesos básicos para la telecardiología

\begin{tabular}{|c|c|}
\hline \multicolumn{2}{|c|}{ Procesos básicos para la telecardiología } \\
\hline 1. & Ingreso paciente a la entidad de salud \\
\hline 2. & Identificación del paciente \\
\hline 3. & $\begin{array}{l}\text { Aceptación del procedimiento por parte del pa- } \\
\text { ciente. }\end{array}$ \\
\hline 4. & $\begin{array}{l}\text { Almacenamiento en el EHR de exámenes de ru- } \\
\text { tina }\end{array}$ \\
\hline 5. & Preparación del paciente para el procedimiento \\
\hline 6. & Captura de la Imágenes de ECG \\
\hline 7. & $\begin{array}{l}\text { Almacenamiento de imágenes en base de datos } \\
\text { por paciente. }\end{array}$ \\
\hline 8. & $\begin{array}{l}\text { Administración de los datos e imágenes (consul- } \\
\text { tas transmisión e impresión de imágenes). }\end{array}$ \\
\hline 9. & $\begin{array}{l}\text { Asignación de consulta previa al paciente para } \\
\text { evaluación de exámenes. }\end{array}$ \\
\hline 10. & Videoconferencia Especialista-Medico general \\
\hline 11. & $\begin{array}{l}\text { Integración del diagnóstico presuntivo, exámenes } \\
\text { de rutina y ECG para decidir si es necesario repe- } \\
\text { tir algún estudio o realizar algún otro extra. }\end{array}$ \\
\hline 12. & $\begin{array}{l}\text { Diagnóstico profesional que incluye el tratamiento } \\
\text { y seguimiento que se le dará. }\end{array}$ \\
\hline 13. & Actualización de información en el registro clínico \\
\hline 14. & Planeación nueva consulta \\
\hline
\end{tabular}

Fuente: tomada de [28].
Tabla 4. Servicios y aplicaciones simulados en la red

\begin{tabular}{|l|l|}
\hline Especialidad & Aplicaciones \\
\hline \multirow{2}{*}{ Registro clínico electrónico } & $\begin{array}{l}\text { Transferencia de archivos } \\
\text { de texto e imágenes }\end{array}$ \\
\hline \multirow{2}{*}{ Tele-cardiología } & Audio consulta \\
\cline { 2 - 2 } & Video consulta \\
\cline { 2 - 2 } & $\begin{array}{l}\text { Transferencia de archivos } \\
\text { de texto e imágenes }\end{array}$ \\
\hline
\end{tabular}

Fuente: elaboración propia.

En la tabla 4 se muestran las aplicaciones que involucra cada una de las especialidades seleccionadas: Registro clínico electrónico y telecardiología.

\section{ANÁLISIS DE REQUERIMIENTOS DISEÑO DE LA RED}

Una vez definidos los servicios para realizar el diseño de la red, es importante definir los requerimientos de red de cada uno de estos servicios, y definir un escenario de red para realizar el análisis correspondiente. El diseño de la red requiere que la red conecte la sede central de la Universidad Militar Nueva Granada (Calle 100 con carrera 11) con la sede de Medicina ubicada en el Hospital Militar Central (Transversal 3a No. 49-00) y, a su vez, soporte los servicios de teleconsulta y telemetría, con las especialidades de registro clínico electrónico y telecardiología respectivamente. A continuación, se describen los requisitos de los servicios, se propone una topología que soporte dichos servicios mediante tecnologías ópticas y, posteriormente, se evalúa mediante ciertos parámetros previamente seleccionados.

\subsection{Requerimientos técnicos de las aplicacio- nes de telemedicina}

En la tabla 5 se muestran los requerimientos mínimos de transmisión, necesarios para el funcio- 


\section{investigación}

Tabla 5. Requerimientos de red de aplicaciones en telemedicina

\begin{tabular}{|l|l|c|c|l|}
\hline Aplicación Multimedia & Descripción & $\begin{array}{c}\text { Aplicación en } \\
\text { tiempo real }\end{array}$ & $\begin{array}{c}\text { Capacidad de } \\
\text { canal }\end{array}$ & \multicolumn{1}{c|}{ Latencia } \\
\hline Audio Consulta & Full Duplex G.729 Audio & Sí & $10-128 \mathrm{Kbps}$ & $\begin{array}{l}\text { Aceptable } \\
<150 \mathrm{~ms} \\
\text { Máximo admisible } \\
400 \mathrm{~ms}\end{array}$ \\
\hline Video Consulta & Half Duplex H.263 video & Si & $64-1920 \mathrm{Kbps}$ & $\begin{array}{l}\text { Aceptable } \\
\text { Máximo admisible } \\
400 \mathrm{~ms}\end{array}$ \\
\hline $\begin{array}{l}\text { Transferencia de exáme- } \\
\text { nes médicos }\end{array}$ & $\begin{array}{l}\text { Transmisión de archivos de tex- } \\
\text { to e imágenes para consulta }\end{array}$ & No & 2 Mbps & - \\
\hline
\end{tabular}

Fuente: tomada de [29] y [30].

namiento de las aplicaciones de audio consulta, video consulta y transferencia de archivos, según las recomendaciones proporcionadas en [29], y según la recomendación de la ITU G.1010 [30]. Los servicios de teleconsulta y telemetría generan tráfico de audio, datos y video.

En la tabla 6 se muestran los requerimientos de la capacidad mínima de canal demandado por las especialidades de registro clínico y tele-cardiología. La capacidad de canal requerida por la teleconsulta es de $256 \mathrm{Kbps}$ por usuario, mientras que la capacidad de canal requerida por la telecardiología es de $896 \mathrm{Kbps}$ por usuario [31].

\subsection{Variables de diseño}

Para evaluar la eficiencia y el comportamiento de la red óptica propuesta, se analizan algunas variables que definen el rendimiento y los parámetros de calidad de servicio que se presentan en la red, como son: Throughput, retardo, Jitter y latencia de la red, soportando los servicios y aplicaciones descritos en la sección anterior.

Tabla 6. Requerimientos de canal para especialidades en telemedicina

\begin{tabular}{|c|c|c|c|c|c|}
\hline $\begin{array}{c}\text { Servicio } \\
\text { de telemedicina }\end{array}$ & Características & $\begin{array}{c}\text { Tipo } \\
\text { de información }\end{array}$ & $\begin{array}{c}\text { Tamaño } \\
\text { de paquetes }\end{array}$ & \multicolumn{2}{|c|}{$\begin{array}{l}\text { Capacidad de canal } \\
\text { requerida }\end{array}$} \\
\hline $\begin{array}{l}\text { Registro Clínico } \\
\text { Electrónico }\end{array}$ & $\begin{array}{l}\text { Digitalización electrónica del histo- } \\
\text { rial en papel del paciente con datos } \\
\text { administrativos, clínicos, etc. }\end{array}$ & $\begin{array}{l}\text { Texto, Datos en for- } \\
\text { mato .txt, .doc, .jpg } \\
\text { o.pdf }\end{array}$ & $1 \mathrm{MB}$ & \multicolumn{2}{|c|}{$256 \mathrm{KBps}$} \\
\hline \multirow{3}{*}{ Telecardiología } & $\begin{array}{l}\text { Adquisición y envío de señales bio- } \\
\text { médicas (ECG) }\end{array}$ & $\begin{array}{l}\text { Texto (Archivos de } \\
\text { texto plano) }\end{array}$ & $100 \mathrm{~KB}$ & $\begin{array}{l}\text { datos: } \\
256 \mathrm{KBps}\end{array}$ & \multirow{3}{*}{$\begin{array}{l}896 \\
\text { KBps }\end{array}$} \\
\hline & Audio conferencia & $\begin{array}{l}\text { Audio digital com- } \\
\text { primido G.729 }\end{array}$ & - & $\begin{array}{l}\text { audio: } \\
128 \mathrm{KBps}\end{array}$ & \\
\hline & videoconferencia & Video digital H.263 & - & $\begin{array}{l}\text { video: } \\
512 \text { KBps }\end{array}$ & \\
\hline
\end{tabular}

Fuente: tomada de [31]. 


\subsubsection{Throughput}

Según la norma RFC 1242, el Throughput se define como la máxima velocidad de transmisión lograda en un enlace, sin que se produzcan descartes de tramas en el dispositivo [32]. Puede representarse por la ecuación (1) y generalmente se expresa en Mbps.

$$
\text { Throughput }=\frac{L}{t}
$$

Donde L representa la Carga de usuario y t, el tiempo de transmisión en segundos.

\subsubsection{Latencia}

La norma RFC 1242 define la latencia como el intervalo de tiempo desde que el último bit de la trama de entrada alcanza el puerto de entrada, hasta que el primer bit de la trama de salida llega al puerto de salida [32], [33]. La diferencia en tiempo entre el instante en el que un dato es recibido y el instante en el que fue enviado. Una función práctica para determinar la latencia en una red está dada por la ecuación (2) [34]:

$$
L=(1 / T)+(P / W)
$$

Donde T hace referencia al Throughput de la red expresado en paquetes por segundo, $\mathrm{P}$ es el tamaño del paquete en Kilobytes/Paquete y W es la capacidad de canal en Kilobytes por segundo.

\subsubsection{Jitter}

El Jitter está determinado por la variación en tiempo desde el momento en el que se supone que un paquete llega, y el tiempo en el que llega realmente. El Jitter es causado por problemas en las redes, como la congestión de la red y la pérdida de sincronización [35]. El Jitter está dado por la ecuación (3) [36].

$$
J_{(i)}=J_{(i-1)}+\frac{D_{(i-1, i)}-J_{(i-1)}}{16}
$$

Donde $\mathrm{J}_{(\mathrm{i})}$ representa la media del Jitter del i-ésimo paquete, $\mathrm{J}_{(\mathrm{i}-1)}$ es la media del Jitter del paquete (i-1), y $\mathrm{D}_{(\mathrm{i}-1 ; \mathrm{i})}$ corresponde a la demora entre el i-ésimo paquete y el paquete (i-1).

\section{RED DE TELEMEDICINA PROPUESTA}

Ya definidos los requerimientos de los servicios de telemedicina, se define un escenario de acuerdo a los requisitos de distancia, conectividad y funcionalidad. En la figura 3 se muestra la localización de los dos puntos finales de la red óptica de telemedicina, los cuales son dos sedes de la Universidad Militar Nueva Granada (sede Calle 100 y sede Facultad de Medicina). La red está compuesta por un canal óptico con una distancia de 6 Kilómetros aproximadamente.

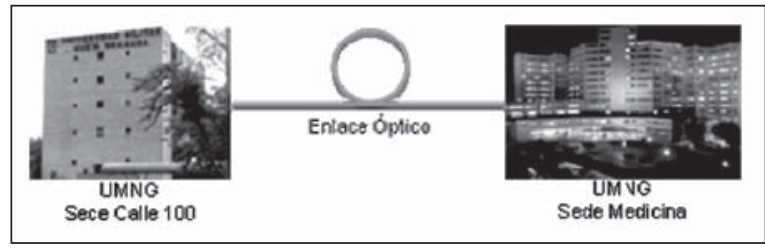

Figura 3. Red para telemedicina

Fuente: elaboración propia.

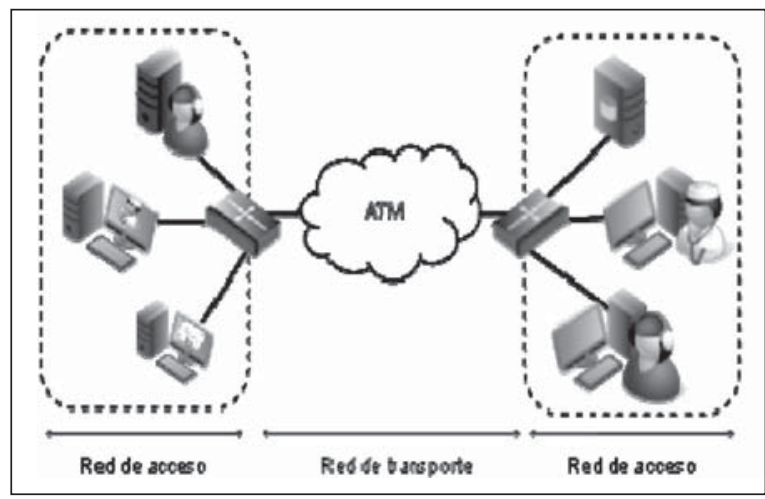

Figura 4. Arquitectura de red para telemedicina

Fuente: elaboración propia. 
Desde el punto de vista de la arquitectura de la red, ésta se compone de una red de una red troncal y dos redes de acceso, ubicadas en cada una de las sedes nombradas anteriormente. La figura 4 muestra la topología de la red con cada una de sus sedes.

Para determinar la capacidad de canal aproximada, necesaria para la implementación de los servicios de telecardiología y teleconsulta, se considera el caso en el que los usuarios se encuentran ocupando el canal simultáneamente:

$B W_{1}=(256 K B p s) * 1=286 K B p s$

$B W_{2}=(896 K B p s) * 2=1792 K B p s$

$B W_{3}=256 K B p s+1792 K B p s=2048 K B p s$

Donde $B W_{1}$ representa la capacidad de canal de la teleconsulta y $B W_{2}$ la capacidad de canal de la telecardiología con dos usuarios, y $B W_{3}$ la capacidad de canal total que resulta ser de 2048 KBps.

En la figura 5 se muestra la red de telemedicina en las dos sedes de la Universidad Militar. En cada una de las sedes hay usuarios del servicio de telemetría, en la sede central de la Universidad Militar hay un usuario de tele consulta, y en la sede de Medicina se encuentra el servidor de base de datos. En cada uno de los extremos de la red se encuentra un nodo encargado de las funciones de conmutación entre los usuarios finales y la red óptica de transporte. Los usuarios de la telemetría utilizan equipos de cómputo para conectarse a la red.

El canal óptico tiene una capacidad de transmisión de 4 MBps., y los equipos de trabajo de los usuarios se conectan a la red mediante cables UTP categoría 510 BaseT.

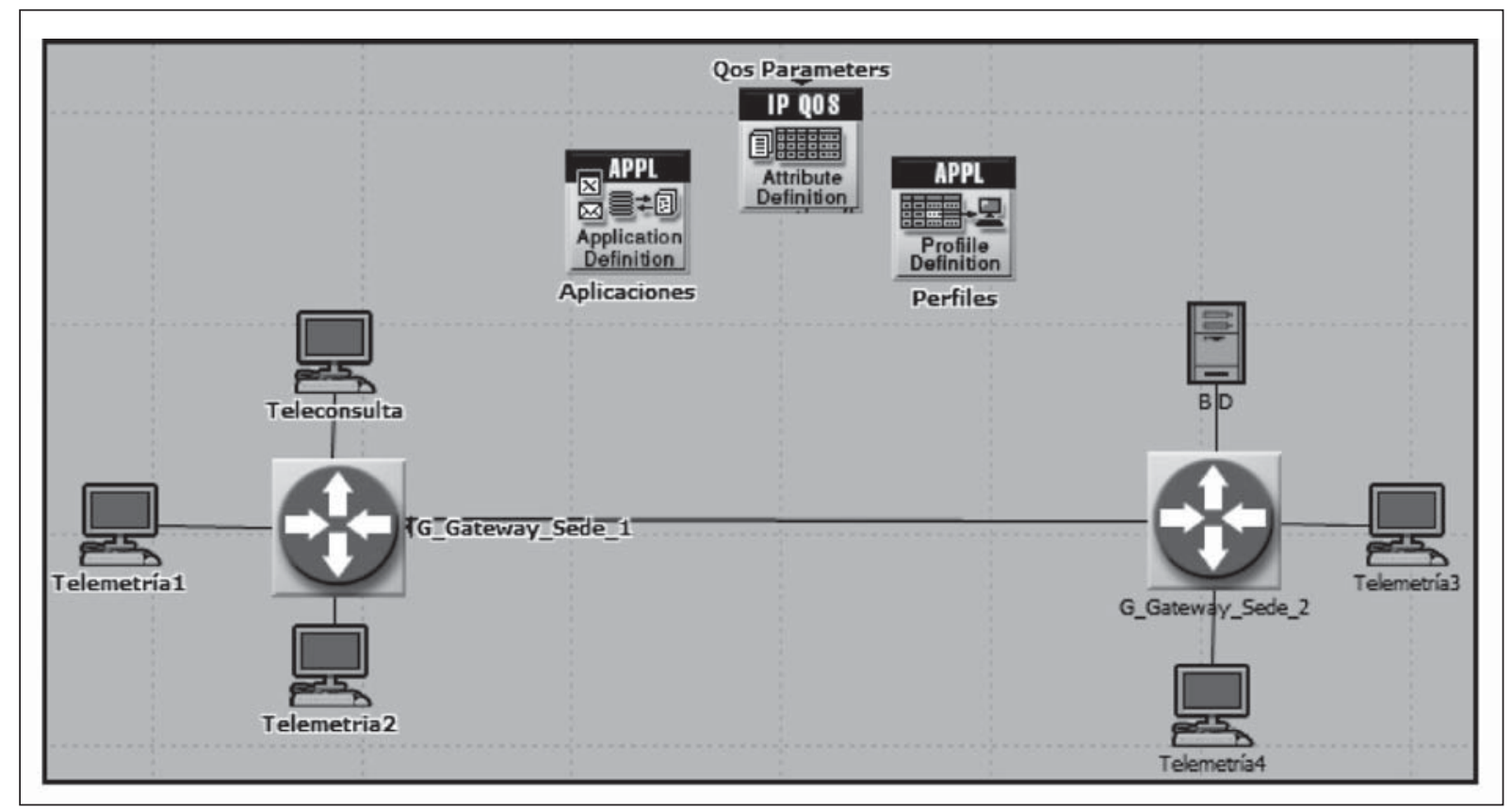

Figura 5. Configuración de red para telemedicina

Fuente: elaboración propia. 


\section{investigación}

\subsection{Simulación de la red}

Las pruebas realizadas consistieron en la simulación las aplicaciones de video consulta, tele consulta y transferencia de archivos entre médicos y pacientes ubicados en ambos extremos de la red, para conseguir datos estadísticos del comportamiento del tráfico generado.

Para simular el funcionamiento de las redes, se utilizó la herramienta OPNET Modeler (Optimized Network Engineering Tool) la cual proporciona un entorno de desarrollo completo para la simulación específica y el análisis de rendimiento de redes de comunicaciones y además, ofrece herramientas para poder adecuar los parámetros de las redes y de cada uno de las aplicaciones necesarias para cada servicio y así obtener datos estadísticos de su comportamiento.

En la red de telemedicina, el tráfico consta de tres componentes: voz, video y datos. El tráfico de voz es generado por aplicaciones de audio conferen- cia mediante aplicaciones de voz sobre IP con una calidad alta de voz, utilizando el estándar G.729 para el audio. El tráfico de video se genera por la aplicación de videoconferencia, video con resolución de 128x240 pixeles a una tasa de grabación y reproducción de 15 frames/sec. El tráfico de datos consiste en la transferencia de archivos con tamaño de $1 \mathrm{MB}$ entre los equipos de médicos y usuarios.

El tráfico de la red se clasifica en dos tipos: tráfico de tasa variable (VBR), para aplicaciones multimedia que requieren transmisión en tiempo real; y tráfico de tasa constante (CBR), para aplicaciones con tráfico continuo o que no son en tiempo real.

El tráfico de la aplicación de transferencia de archivos es de tipo continuo y depende del tamaño de los paquetes y del tiempo en el que la aplicación realiza repeticiones de envío. El tráfico de las aplicaciones de video consulta y teleconsulta tienen una distribución estadística exponencial según el estudio realizado en [37] y [38]. En la tabla 7 se muestran los parámetros configurados

Tabla 7. Parámetros de tráfico para la simulación

\begin{tabular}{|c|c|c|c|}
\hline Especialidad & $\begin{array}{l}\text { Aplicaciones } \\
\text { Requeridas }\end{array}$ & $\begin{array}{l}\text { Perfiles configu- } \\
\text { rados en OPNET }\end{array}$ & Parámetros configurados en OPNET \\
\hline \multirow[t]{2}{*}{$\begin{array}{l}\text { Registro clínico } \\
\text { electrónico }\end{array}$} & $\begin{array}{l}\text { Transferencia de ar- } \\
\text { chivos de texto e } \\
\text { imágenes }\end{array}$ & $\begin{array}{l}\text { FTP (File Transfer } \\
\text { Protocol) }\end{array}$ & $\begin{array}{l}\square \text { Tamaño de paquete=Distribución de probabilidad } \\
\text { constante (media } 1 \mathrm{MB} \text { ) } \\
\square \quad \text { Tiempo entre peticiones=Distribución de probabilidad } \\
\text { constante (media de } 10 \mathrm{~s})\end{array}$ \\
\hline & Base de datos & Base de datos & $\begin{array}{l}\square \text { Tamaño de paquete=Distribución de probabilidad } \\
\text { constante (media } 1 \mathrm{MB})\end{array}$ \\
\hline \multirow{3}{*}{ Tele-cardiología } & Audio consulta & Aplicación de voz & $\begin{array}{ll}\square & \text { Códec } \mathrm{G} .729 \\
\square \quad \text { Intervalos de Silencio= Distribución exponencial (me- } \\
\text { dia } 0,65 \mathrm{~s} \text { ) } \\
\square \quad \begin{array}{l}\text { Intervalos de voz= distribución exponencial (media de } \\
0,352 \mathrm{~s} \text { ) }\end{array}\end{array}$ \\
\hline & Video consulta & $\begin{array}{l}\text { Aplicación } \\
\text { de video }\end{array}$ & $\begin{array}{l}\square \quad \text { Video con resolución de } 352 \times 240 \text { pixeles. } \\
\square \quad \text { Tamaño de trama de streaming: Distribución de pro- } \\
\text { babilidad constante (media } 253,44 \mathrm{~KB} \text { ) } \\
\square \quad \text { Tasa de grabación y reproducción de } 15 \text { frames/sec }\end{array}$ \\
\hline & $\begin{array}{l}\text { Transferencia de ar- } \\
\text { chivos de texto e } \\
\text { imágenes }\end{array}$ & $\begin{array}{l}\text { FTP (File Transfer } \\
\text { Protocol) }\end{array}$ & $\begin{array}{l}\square \text { Tamaño de paquete=Distribución de probabilidad } \\
\text { constante (media } 100 \mathrm{~KB} \text { ) } \\
\square \quad \text { Tiempo entre peticiones=Distribución de probabilidad } \\
\text { constante (media de } 100 \mathrm{~s} \text { ) }\end{array}$ \\
\hline
\end{tabular}

Fuente: tomada de [37] y [39]. 


\section{investigación}

Tabla 8. Casos para simulación de la red

\begin{tabular}{|l|c|c|c|c|c|}
\hline Aplicaciones & Caso 1 & Caso 2 & Caso 3 & Caso 4 & Caso 5 \\
\hline Transferencia de Archivos médicos & & $\mathrm{X}$ & $\mathrm{X}$ & & $\mathrm{X}$ \\
\hline Almacenamiento en Base de Datos & & $\mathrm{X}$ & $\mathrm{X}$ & & \\
\hline Audio Consulta & $\mathrm{X}$ & $\mathrm{X}$ & $\mathrm{X}$ & & \\
\hline Video Consulta & & & $\mathrm{X}$ & $\mathrm{X}$ & $\mathrm{X}$ \\
\hline
\end{tabular}

Fuente: elaboración propia.

para la simulación de la red establecidos según los requerimientos de los servicios de telemedicina que serán objeto de estudio.

\section{ANÁLISIS DE RESULTADOS}

Una vez configurados los parámetros, se realizan las simulaciones con duración de 12 horas y, a partir de ellas, se obtienen los resultados que permiten determinar algunas características importantes de rendimiento de la red.

A continuación se muestran los resultados obtenidos en distintas simulaciones de la red, en las que se evaluó la afectación en el rendimiento que genera cada aplicación, como se muestra en la ta- bla 8 , y se definen algunos casos sobre los cuales estarán sustentadas las simulaciones:

Una vez comprobado que el rendimiento de una red óptica es mucho mejor que el de una red tradicional Ethernet. Las simulaciones están concentradas en el análisis de la red óptica, evaluando cada uno de los servicios con sus aplicaciones a partir de las variables definidas anteriormente: throughput, jitter y latencia.

\subsection{Throughput}

La figura 6 muestra el resultado obtenido de la medición del Throughput en la red troncal, con las aplicaciones de los servicios de EHR y telecardiología.

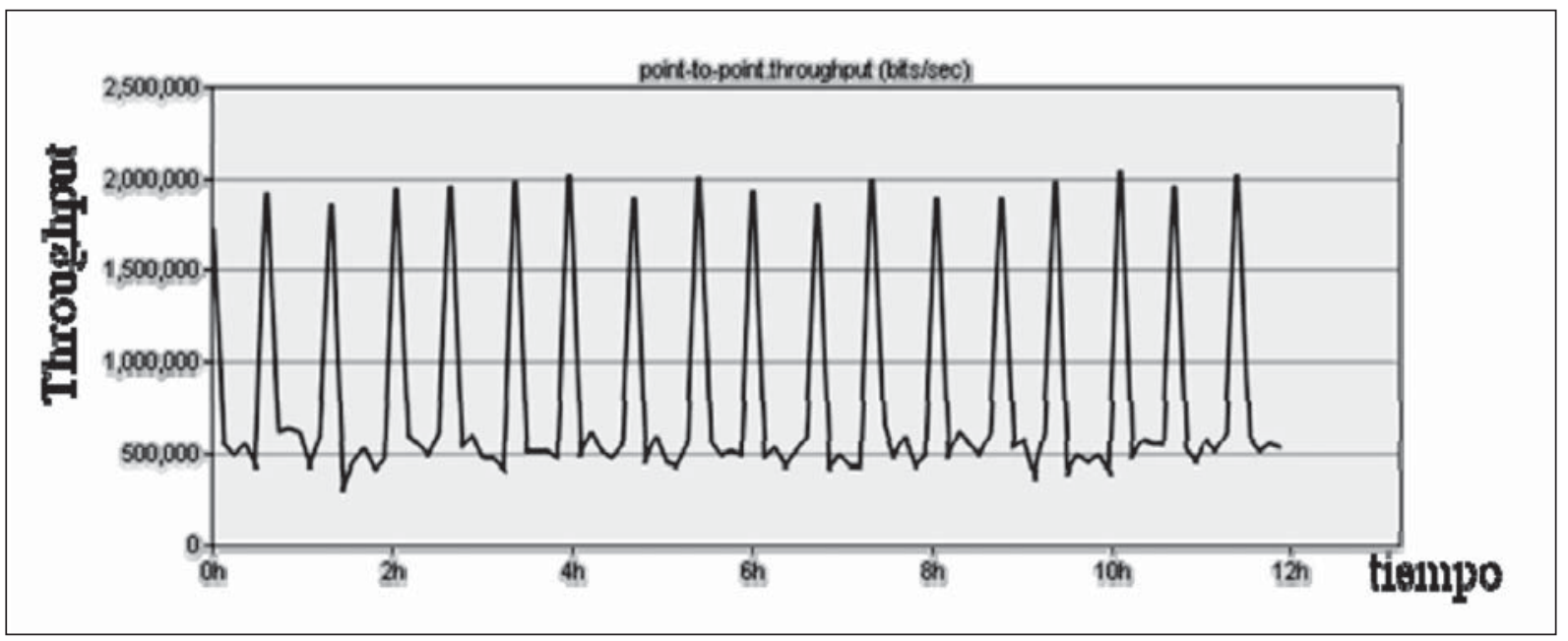

Figura 6. Throughput en simulación de EHR y telecardiología

Fuente: elaboración propia. 


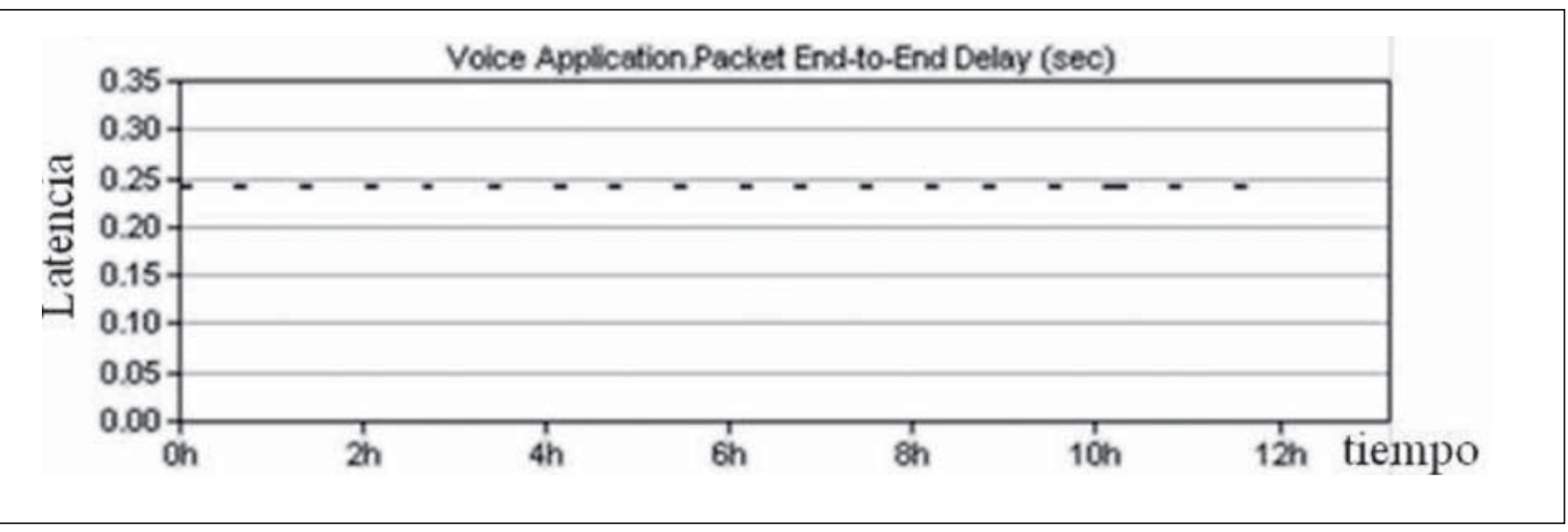

Figura 7. Latencia en aplicación de audio consulta, Caso 1

Fuente: elaboración propia.

\subsection{Latencia}

En las figuras 7, 8 y 9, se puede apreciar el resultado la latencia de la aplicación de audio consulta, así mismo, en la tabla 9 se muestra el promedio de la latencia obtenida por la simulación. La latencia en la aplicación de audio consulta fue medida en los siguientes casos $(1,2,3)$ :

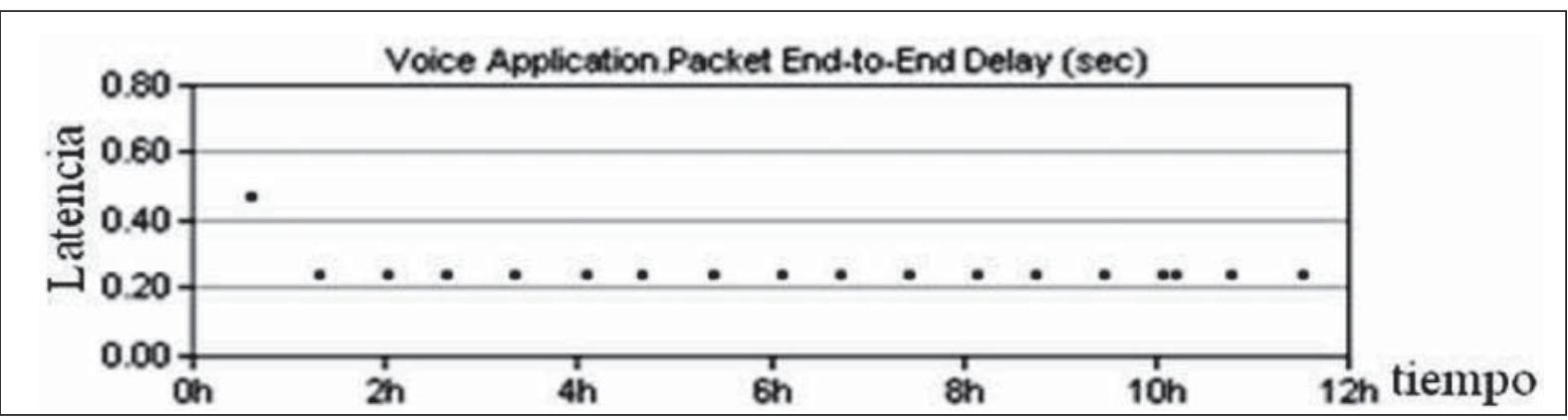

Figura 8. Latencia en aplicación de audio consulta, Caso 2

Fuente: elaboración propia.

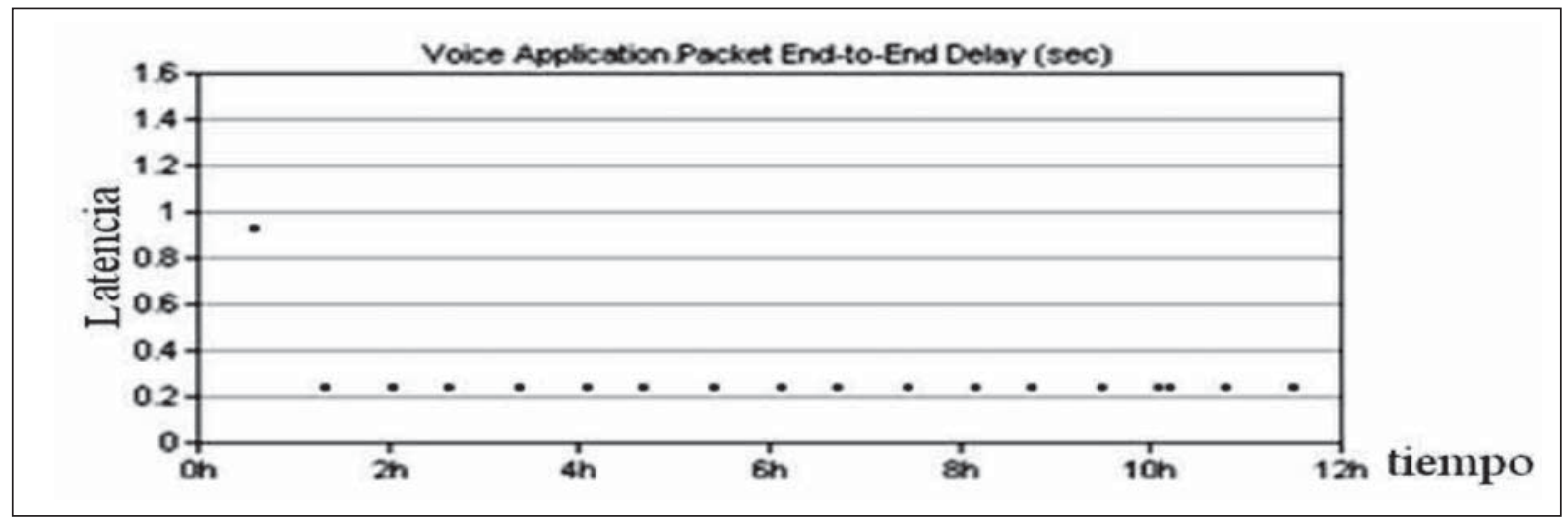

Figura 9. Latencia en aplicación de audio consulta, Caso 3

Fuente: elaboración propia. 


\section{investigación}

Tabla 9. Latencia en simulación de audio consulta

\begin{tabular}{|c|c|c|c|}
\hline $\begin{array}{c}\text { Latencia } \\
\text { Audio } \\
\text { Consulta }\end{array}$ & Caso 1 & Caso 2 & Caso 3 \\
\cline { 2 - 4 } & $240,3 \mathrm{~ms}$ & $312 \mathrm{~ms}$ & $354 \mathrm{~ms}$ \\
\hline
\end{tabular}

Fuente: elaboración propia.

En las figuras 10, 11 y 12, se puede apreciar el resultado la latencia de la aplicación de video consulta, medida en los siguientes casos y, así mismo, en la tabla 10 se resume la latencia pro- medio determinada por simulación. En el primero de ellos se simuló el comportamiento de la red únicamente con la aplicación de video consulta. En el segundo caso, se simuló el comportamiento de la red con la aplicación de video consulta y las aplicaciones de la especialidad del registro clínico electrónico (transferencia de archivos y base de datos). Finalmente, el en tercer caso la simulación de la red incluyó las aplicaciones de video consulta, audio consulta y las aplicaciones del registro clínico electrónico.

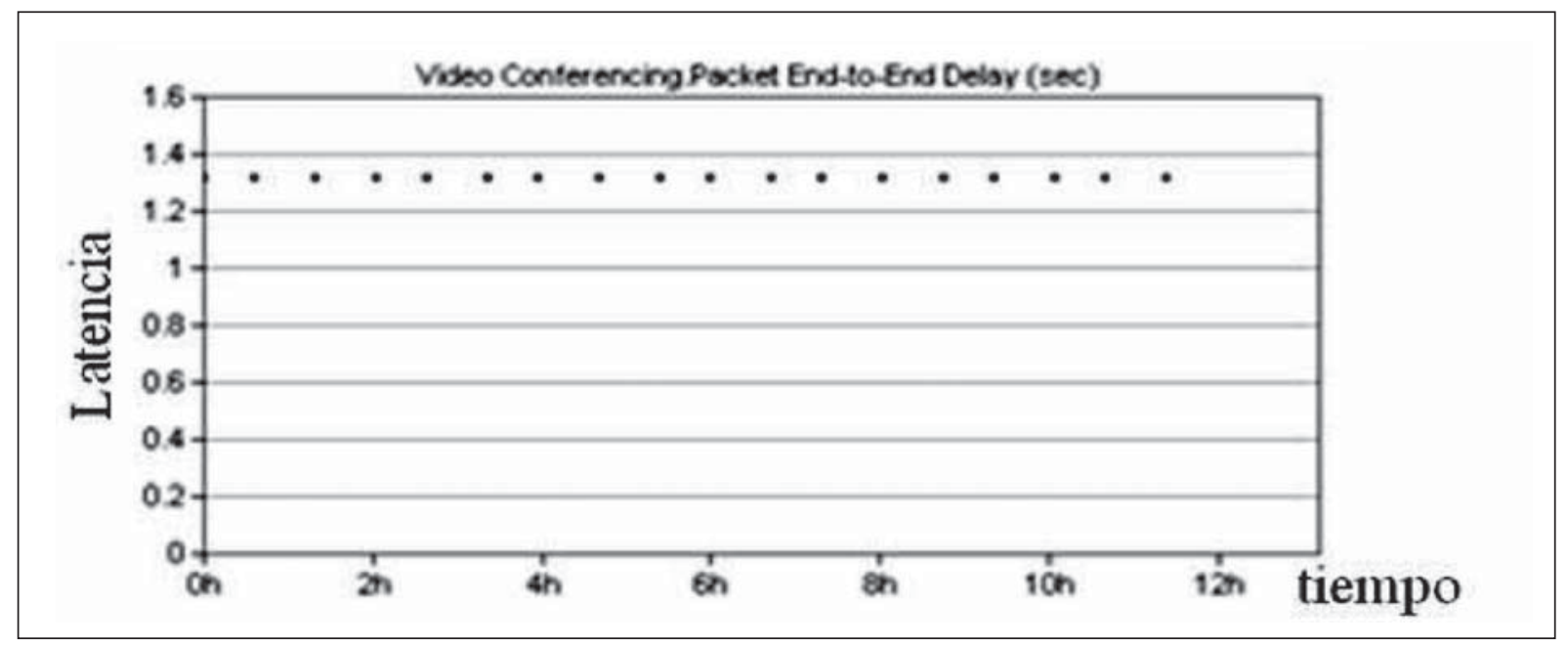

Figura 10. Latencia en aplicación de video consulta, Caso 1

Fuente: elaboración propia.

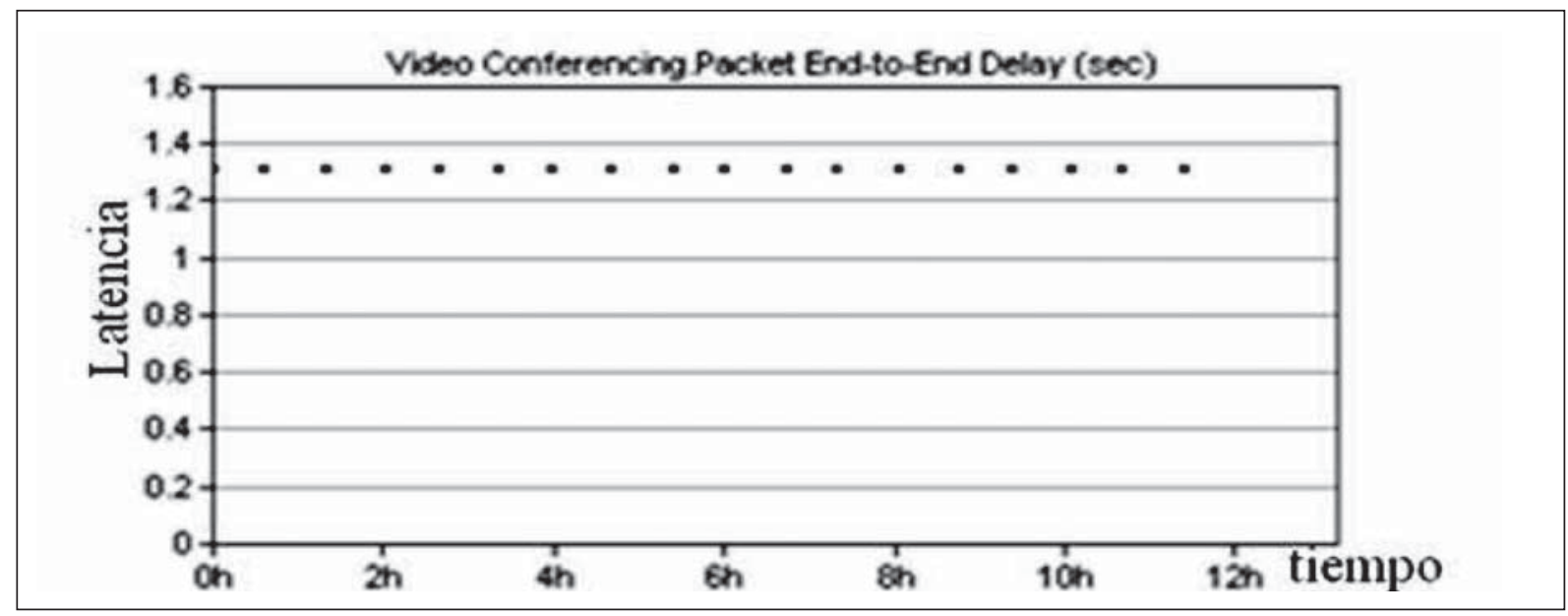

Figura 11. Latencia en aplicación de video consulta, Caso 2

Fuente: elaboración propia. 


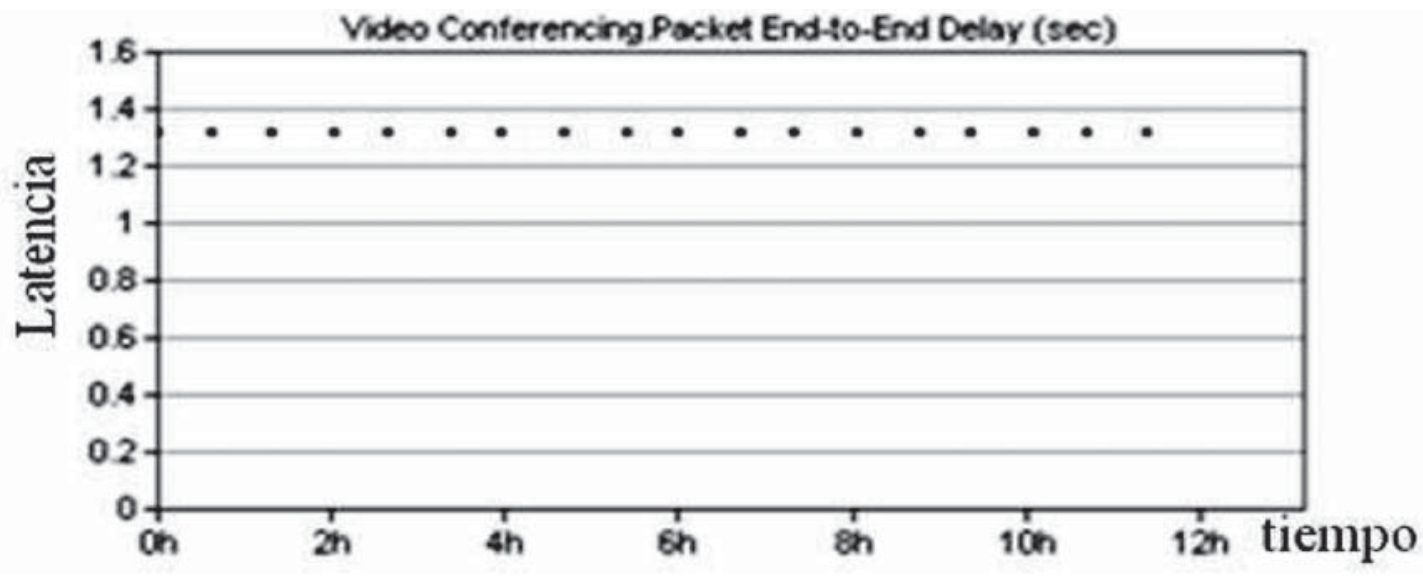

Figura 12. Latencia en la aplicación de video consulta, Caso 3

Fuente: elaboración propia.

Tabla 10. Latencia en simulación de video consulta

\begin{tabular}{|c|c|c|c|}
\hline $\begin{array}{c}\text { Latencia } \\
\text { Audio } \\
\text { Consulta }\end{array}$ & Caso 4 & Caso 5 & Caso 3 \\
\cline { 2 - 4 } & $1,3159 \mathrm{~s}$ & $1,3152 \mathrm{~s}$ & $1,31595 \mathrm{~s}$ \\
\hline
\end{tabular}

Fuente: elaboración propia.

\subsection{Jitter}

Las figuras 13, 14 y 15, muestran el Jitter obtenido en la aplicación de audio consulta en los mismos casos dispuestos en la sección 7.2 y se resume su promedio en la tabla 11.

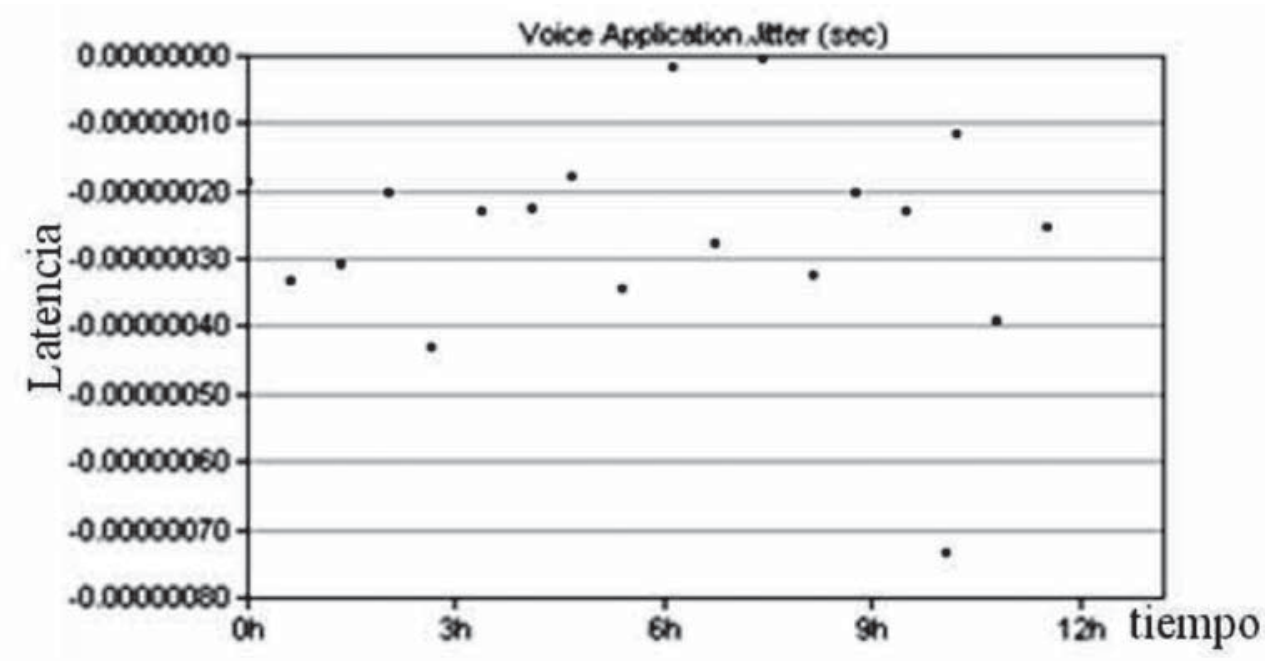

Figura 13. Jitter en aplicación de audio consulta, Caso 1 Fuente: elaboración propia. 


\section{investigación}

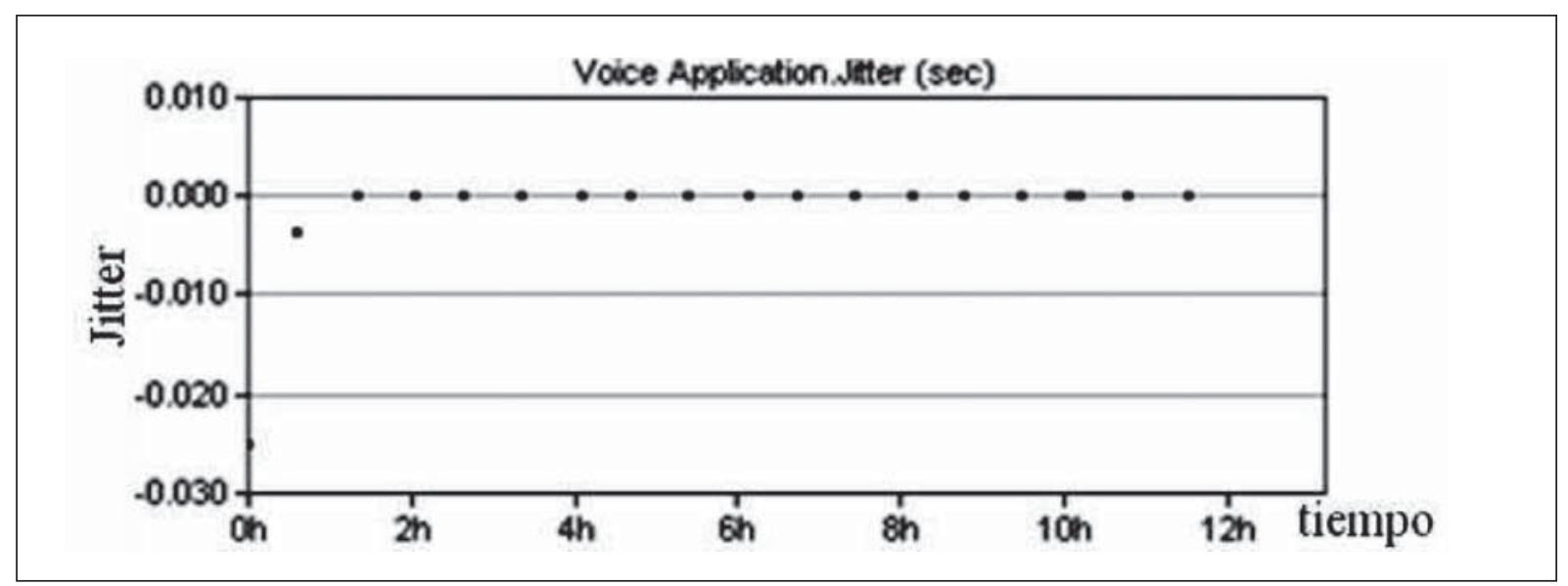

Figura 14. Jitter en aplicación de audio consulta, Caso 2

Fuente: elaboración propia.

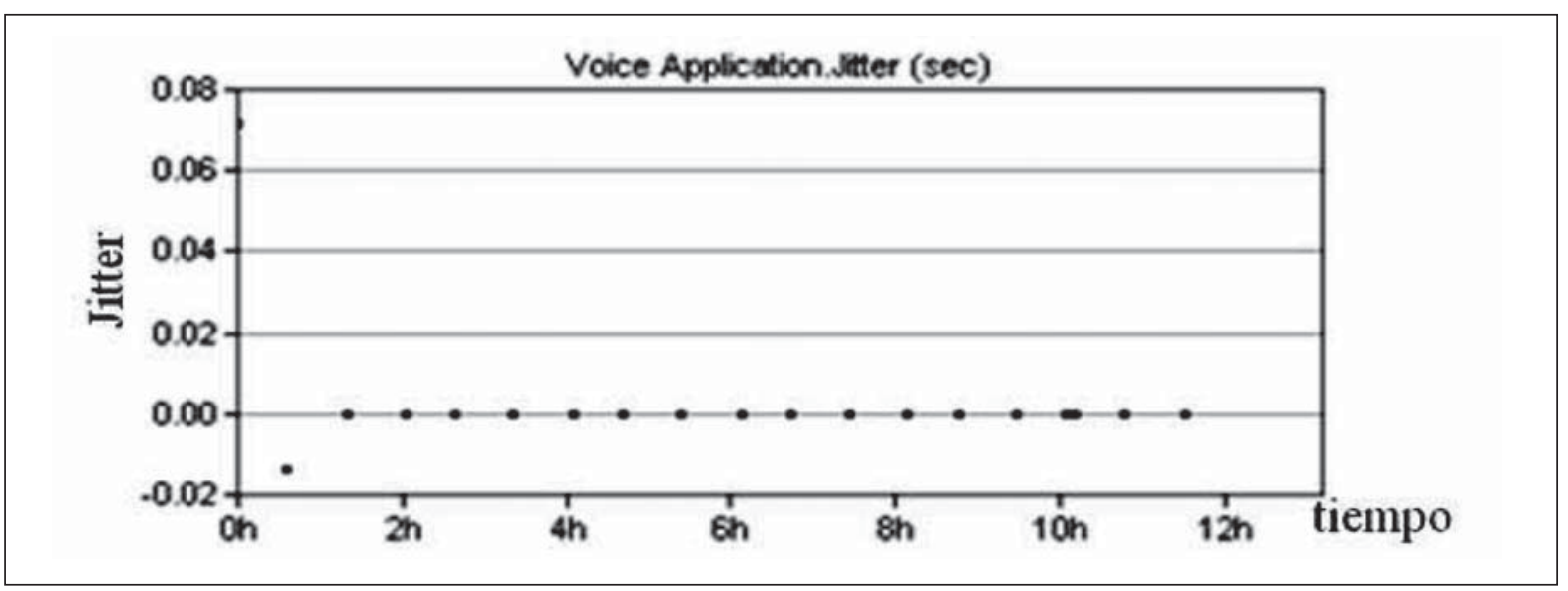

Figura 15. Jitter en aplicación de audio consulta, Caso 3

Fuente: elaboración propia.

Tabla 11. Jitter en simulación de audio consulta

\begin{tabular}{|c|c|c|c|}
\hline Jitter & Caso 1 & Caso 2 & Caso 3 \\
\hline consulta & $-0,00026 \mathrm{~ms}$ & $-1,5 \mathrm{~ms}$ & $3,03 \mathrm{~ms}$ \\
\hline
\end{tabular}

Fuente: elaboración propia.

Teniendo en cuenta que el valor máximo admisible del Jitter para voz (G.729) es de 200 ms [40].

En los tres casos, los valores del Jitter cumplieron con el valor de umbral establecido para una óptima calidad de las sesiones de audio consulta.

\section{CONCLUSIONES}

En esta investigación, se ha propuesto una red para servicios de telemedicina con una arquitectura óptica, la cual demostró que es posible cumplir con los requerimientos de calidad de servicio de algunas aplicaciones, como lo son: audio consulta y registro clínico electrónico EHR.

El análisis de los resultados de las simulaciones de la red, demuestra que, aunque los niveles de latencia en la aplicación de audio consulta se en- 


\section{investigación}

cuentran por debajo del valor admisible de latencia para voz, también se encuentran por encima del valor aceptable, lo que no garantiza el cumplimiento de la calidad del servicio en audio consulta. Sin embargo, los valores del Jitter mostraron un óptimo desempeño de la red con la aplicación de audio consulta en los casos estudiados, incluso se obtuvieron valores negativos de Jitter, lo que supone una llegada de los paquetes con un intervalo de tiempo menor a la diferencia de tiempo con que fueron enviados.

Es importante resaltar la evaluación de una red óptica sobre estos dos puntos, permitiendo ver una aproximación del comportamiento de la misma para pensar en una implementación real, y ver las ventajas de una red óptica sobre una red convencional.
Se recomienda que, para futuras investigaciones relacionadas, se modifiquen las características de los dispositivos y conexiones finales, así como de las características de la red troncal para mejorar el desempeño de la red para las aplicaciones mencionadas junto con la simulación de la mayoría de los servicios de telemedicina.

\section{FINANCIACIÓN}

El financiamiento y aval para el desarrollo del proyecto de investigación que dio como parte de sus resultados el presente artículo fue hecho por la Universidad Militar Nueva Granada.

\section{REFERENCIAS}

[1] R. Komiya, A proposal for telemedicine reference model for future standardization, [Memorias del evento] 7thInternational Workshop HEALTHCOM2005, junio, 2005.

[2] A. Shaikh, Asadullah and M. Misbahuddin, A system design for a tele-medicine health care system, [Tesis de Maestría en Ingeniería de Software y Gestión], IT University of Goteborg Gôteborg, Suiza, 2007.

[3] E. Putra, E. Supriyanto, J. Din and H. Satria, Cross Layer Design of Wireless LAN for Telemedicine Application, [Memorias del evento], Third Asia International Conference on Modelling \& Simulation. Bandung, Indonesia, mayo, 2009.

[4] B. Singh, M. Rahman, K. Tapan, L. Abu, and M. Bashar, "Channel Based Simulation and Analysis of IEEE802.16 (WiMAX) - 2004IW MAN-OFDM Physical
Layer for BWA to Support Telemedicine", in International Conference on Complex Medical Engineering (CME), 2010 IEEE/ ICME, Gold Coast, Australia. 2010.

[5] L. Qiao and P. Koutsakis, "Fair and Efficient Scheduling for Telemedicine Traffic Transmission over Wireless Cellular Networks", IEEE $69^{\text {th }}$ Vehicular Technology Conference, VTC 2009, Barcelona, España, abril, 2005.

[6] E. Husni, Y. Heryadi, W. Woon, M. Arifianto, D. Viswacheda and L. Barukang, "Mobile ad hoc network and mobile IP for future mobile telemedicine system", in International Conference on Wireless and Optical Communications Networks, 2006 IFIP. Bangalore, India, abril, 2006.

[7] R. Ramaswami and K. N. Sivarajan, $O p$ tical Networks: A Practical Perspective. 
Segunda Edición. San Francisco, USA: Morgan Kaufmann Publishers, 2002.

[8] J. Campany y B. Ortega, Redes Ópticas. Valencia, España: Editorial de la UPV. 2006.

[9] R. Ramaswami and K. N. Sivarajan, $O p$ tical Networks: A Practical Perspective, Segunda Edición. San Francisco, USA: Morgan Kaufmann Publishers. 2002.

[10] N. Kashima, Passive Optical Components for Optical Fiber Transmission, Boston, USA: Artech House Publisher. 1995.

[11] M. Alban, Metodología de diseño de redes de fibra Óptica, [Tesis], Escuela Politécnica del Ejército, Sangolquí, Ecuador, 2006.

[12] B. Mukherjee, Optical WDM Networks, New York, USA: Ed. Springer Science \& Business Media. 2006.

[13] M. Y. Akhtar, The Handbook of Optical Communication Networks, Florida, USA. Editorial Mohammad, 2003.

[14] M. C. Necker, C. M. Gauger and S. Bodamer, "A new efficient integrated routing scheme for SDH/SONET-WDM multilayer networks", in Optical Fiber Communication Conference, Optical Society of America. 2003.

[15] Network node interface for the synchronous digital hierarchy (SDH), Recomendación ITU-T G.707, International Telecommunication Union - ITU-, 2007.

[16] Telecommunications - Synchronous Optical Network (SONET), Estándar ANSI T1.105, American National Standards Institute. 2001.
[17] M. C. Necker, C. M. Gauger and S. Bodamer, "A new efficient integrated routing scheme for SDH/SONET-WDM multilayer networks", in Optical Fiber Communication Conference, Optical Society of America, 2003.

[18] Network node interface for the synchronous digital hierarchy (SDH). Recomendación ITU-T G.707, International Telecommunication Union (ITU), 2007.

[19] Telecommunications - Synchronous Optical Network (SONET), Estándar ANSI T1.105, American National Standards Institute (ANSI), 2001.

[20] Network node interface for the synchronous digital hierarchy (SDH), Recomendación G.707/Y.1322 (2007) Amendment 2 (11/09). ITU-T G.707, International Telecommunication Union (ITU), 2009.

[21] General description of asynchronous transfer mode, Recomendación ITU-T I.150, International Telecommunication Union (ITU), 1992.

[22] Kien Du Phung and Antonio R. Dimalanta Jr., Study of a SONET STS-3c based ATM User Network Interface Design, [Memorias del evento], Sixth Annual IEEE International ASIC Conference and Exhibit, Rochester, USA. 1993.

[23] J. Wallauer; A. Wangenheim, R. Andrade and Douglas D. J. de Macedo, "A Telemedicine Network Using Secure Techniques and Intelligent User Access Control", [Memorias del evento], 21st IEEE International Symposium on ComputerBased $\mathrm{Me}$ dical Systems. Maribor, Slovenia, junio, 2007. 


\section{investigación}

[24] R. Komiya, "A proposal for telemedicine reference model for future standardization", [Memorias del evento], 7th International Workshop Healthcom 2005. Busan, Korea, junio, 2005

[25] A. Shaikh, Asadullah and M. Misbahuddin, A system design for a tele-medicine health care system, [Tesis de Maestría en Ingeniería de Software y Gestión]. IT University of Goteborg Gôteborg, Suiza, 2007.

[26] Organización Panamericana de la Salud OPS/OMS, ORAS-CONHU Organismo Andino de Salud, "Aplicaciones de Telecomunicaciones en la salud en la Subregión Andina”, en Serie Documentos Institucionales, 2000.

[27] T.M. Fliedner, M. Weiss, H. P. Grossmann, B. Pieper, A. V. Akleyev, and T. A. Varfolomeyeva, "Teleconsultation in Radiation Medicine", in Results of regular telemedicine consultations between the Health Sciences Centre of the University of Ulm, Germany and Urals Research Centre for Radiation Medicine, Chelyabinsk, Russia. 2002.

[28] N. Ozen and B. Karlik, "A telecardiology system design with real-time diagnosis and teleconsultation", [Memorias del evento], First International Conference on Applications of Digital Information and Web Technologies ICADIWT 2008, Ostrava, República Checa. agosto, 2008.

[29] L. Aparicio, I. González, J. Angulo, J. Benifez. Forero, M. J. Duarte y H. E. Cifuentes, "Red urbana de telemedicina para Bogotá operador", [Documento inédito], Grupo de Investigación en Telemedicina GITEM, Universidad Distrital Francisco José de Caldas, Bogotá, Colombia. 2008.
[30] End-user multimedia QoS categories, Recomendación ITU-T G.1010, International Telecommunication Union (ITU), 2001.

[31] C. Arcila y M. Loaiza. "Diseño de un enlace de telemedicina para el Hospital Universitario San Juan de Dios del Quindío", [Tesis Trabajo de grado], Facultad de Ingeniería, Universidad del Quindío. 2010.

[32] S. Bradner, "Benchmarking Terminology for Network Interconnection Devices", in Internet Engineering Task Force (IETF). Estándar RFC 1242, julio 1991

[33] H. Schulzrinne, S. Casner, R. Frederick and V. Jacobson, "RTP: A TransportvProtocol for Real-Time Applications", in Internet Engineering Task Force (IETF). Estándar RFC 3550, Jul. 2003.

[34] D. A. Patterson, "Latency Lags BandWidth", in Comunicado del Association for Computing Machinery (ACM) Magazine (CACM), Octubre, 2004

[35] Hung-Ching Chang, Chun-Chin Chen and Chih-Feng Lin, "XScale Hardware Acceleration on Cryptographic Algorithms for IPSec Applications", [Memorias del evento], International Conference on Information Technology: Coding and Computing, ITCC 2005, Las Vegas USA, Abril, 2005.

[36] H. Schulzrinne, S. Casner, R. Frederick and V. Jacobson, "RTP: A TransportvProtocol for Real-Time Applications", in Internet Engineering Task Force (IETF). Estándar RFC 3550, Jul., 2003.

[37] C. Arcila y M. Loaiza, Diseño de un enlace de telemedicina para el Hospital Universitario San Juan de Dios del Quindio, [Tesis Trabajo de grado], Facultad de Ingeniería Universidad del Quindío, 2010. 


\section{investigación}

[38] I. Martínez, J. Salvador, J. Fernández and J. García, "Traffic requirements evaluation for a Telemedicine network", [Memorias del evento], International Congress on Computational Bioengineering ICCB 2003, Zaragoza, España, 2003.

[39] Coding of speech at $8 \mathrm{kbit} / \mathrm{s}$ using conjugate-structure algebraic-code-excited linear prediction (CS-ACELP), Recomendación ITU-T G.729, International Telecommunication Union (ITU), 1996

[40] E. Kelly, "Quality of Service In Internet Protocol (IP) Networks", Journal of the International Communications Industries Association, Infocomm, Shanghai, China. 2002. 